\title{
Impacto de estrategias en la epidemiología de la pudrición basal, la pudrición carbonosa y el rendimiento de caraota (Phaseolus vulgaris $\mathrm{L}$.)
}

\author{
Impact of management strategies in the basal rot, charcoal rots epidemiology and Phaseolus \\ vulgaris L. yield. \\ Ulacio Osorio Dilcia $^{1 *}$, Jiménez Tamayo María ${ }^{1}$, Perdomo Wilfredo ${ }^{1}$, Moreno Novis ${ }^{2}$
}

\begin{tabular}{l} 
Datos del Artículo \\
\\
\hline${ }^{1}$ Universidad Centroccidental Lisandro \\
Alvarado, Laboratorio de \\
Fitopatología, Estado Lara Venezuela. \\
Apartado 400 3001 Lara. Venezuela \\
Telf. (0251) 259.2490 - 259.2493. \\
Fax. $\quad$ (0251) \\
dilciau@ucla.edu.ve \\
mjimeneztamayo@gmail.com. \\
wilfredoperdomo@yahoo.es \\
namoreno@inia.gob.ve \\
2Instituto Nacional de Investigaciones \\
Agrícolas. Barinas estado Barinas \\
*Dirección de contacto: \\
Universidad Centroccidental Lisandro \\
Alvarado, Laboratorio de Fitopatología \\
Ulacio Osorio Dilcia \\
E-mail address: dilciau@ucla.edu.ve \\
\hline
\end{tabular}

\section{Palabras clave: \\ Tebuconazole, nitrato de calcio, Trichoderma, solarización, Macrophomina phaseolina Sclerotium rolfsii \\ J Selva Andina Res Soc 2012; 3(2):14-26. \\ Historial del artículo \\ Recibido Septiembre, 2012. Devuelto Diciembre 2012 Aceptado Enero, 2013. Disponible en línea, Febrero, 2013

\begin{tabular}{l}
\hline Key words: \\
Tebuconazole, \\
calcium nitrate, \\
Trichoderma, solarization, \\
Macrophomina phaseolina, \\
Sclerotium rolfsii \\
\hline
\end{tabular}

\section{Editado por: \\ "Selva Andina \\ Research \\ Society"}

\section{Resumen}

Se evaluó el efecto de estrategias químicas, físicas, biológicas y culturales solas o combinadas en la epidemiología de la pudrición basal (Sclerotium rolfsii), la pudrición carbonosa (Macrophomina phaseolina) y el rendimiento de Phaseolus vulgaris cv Tacarigua en el estado Barinas, Venezuela. Se aplicó Tebuconazole (Teb) a la semilla (1 L/Ton) y a los 30 y 60 días después de la siembra (1 L/ha), Trichoderma harzianum (Tri) a la semilla (15 g por cada $1.5 \mathrm{k}$ ) y a los 15, 30, 45 y 60 días después de la siembra (30 g/10 L de agua), solarización (Sol) por 15 días y nitrato de calcio (Ca) (60 g/10 L de agua) cada 15 días hasta los 60 días de desarrollo del cultivo. La pudrición basal se registró hasta los 42 días después de la siembra, limitándose a menos de 5.3\% en Teb y en SolTeb, presentándose la mayor incidencia en Tri con 28.5\%, superando al testigo (14.5\%). Posteriormente predominó la pudrición carbonosa enfermando el resto de las plantas, completando el $100 \%$, en todos los tratamientos. No obstante, Teb presentó el mayor rendimiento equivalente a $555 \mathrm{k} / \mathrm{ha}$, siendo estadísticamente diferente al tratamiento TriCa que presentó el menor rendimiento con $31 \mathrm{k} / \mathrm{ha}$, sin embargo, con el fungicida y el Ca, las raíces no formaron nódulos fijadores de nitrógeno. Se concluye que $S$. rolfsii fue sensible a la acción de algunos de los tratamientos; no así M. phaseolina, sin embargo, las plantas fueron capaces de producir semillas aparentemente sanas en tratamientos con menor severidad de la pudrición carbonosa.

2012. Journal of the Selva Andina Research Society. Bolivia. Todos los derechos reservados.

\begin{abstract}
The effect of chemical, physical, biologycal and cultural strategies individually or combinated were evaluated in the epidemiology of the basal rot (Sclerotium rolfsii), charcoal rot (Macrophomina phaseolina) and the Phaseolus vulgaris cv Tacarigua yield at Barinas state from Venezuela. In the experiment, Tebuconazole (Teb) was applicated at seed (1 L/Ton) and at soil, a los 30 y 60 days after of the sow $(1 \mathrm{~L} / \mathrm{ha})$; Trichoderma harzianum (Tri) was applicated at seed ( $15 \mathrm{~g}$ for each $1.5 \mathrm{k}$ ) and to 15, 30, $45 \mathrm{y} 60$ days after of the sow ( $30 \mathrm{~g} / 10 \mathrm{~L}$ of water). On the other hand, soil was solarizated (Sol) during 15 days and calcium nitrate (Ca) (60 g/10 L of water) was applicated each 15 days until 60 days of growth of cultivated plants. Basal rot was registered as far as 42 days after of the sow, showing less of $5.3 \%$ in Teb y the combination SolTeb. The hightest incidence of this disease was observed in the treatment Tri with $28.5 \%$, being highter that control (14.5\%). Last to 42 days predominated the charcoal rot in the rest of the plants for a total of $100 \%$ of incidente in everything the treatments. Nevertheless, Teb showed the hightest yield with $555 \mathrm{k} / \mathrm{ha}$, being different estatistically at treatment TriCa, which showed the lowest yield with $31 \mathrm{k} / \mathrm{ha}$, however, the roots not formed nodules nitrogen uptake in these replications with the fungicide and Ca. It is concluded that $S$. rolfsii was sensible at action of some of the treatments; but not M. phaseolina; nevertheless, the plants were capables to produce seeds health apparently in treatments in which observed less severity of charcoal rot.
\end{abstract}




\section{Introducción}

A nivel mundial, la caraota o frijol negro (Phaseolus vulgaris L.), es una de las once especies vegetales que alimentan a la población humana, adaptándose a casi todos los ambientes (Mora 1997). La calidad y el rendimiento de este cultivo se ven afectado por diversos problemas fitosanitarios. Entre estos, hongos como Sclerotium rolfsii, causante de la pudrición basal y Macrophomina phaseolina, patógeno de la pudrición carbonosa, causan pérdidas importantes ocasionando daños en pre y post emergencia o reduciendo el vigor de las plantas adultas y el rendimiento (Acevedo 2003, Morros 2001).

\section{Sclerotium rolfsii y Macrophomina phaseolina} producen esclerocios en el suelo, siendo el principal propágulo de diseminación en ambos hongos. Estos patógenos son difíciles de controlar por su capacidad de mantenerse viables en el suelo, por largos períodos de tiempo a través de los esclerocios (APS 1975, Punja 1985). S. rolfsii, ha sido señalado a nivel mundial afectando a más de 180 cultivos diferentes, presentándose al inicio o al final del ciclo de cultivo, dependiendo del hospedante (Punja 1985). En el caso de la caraota se ha observado que la enfermedad afecta las primeras etapas fenológicas, desde la semilla. En Venezuela se ha evidenciado en zonas agrícolas de los estados Cojedes, Guárico y Portuguesa (Ramírez et al 1998).

Macrophomina phaseolina también presenta una amplia cantidad de hospedantes (Morros 2001). En países tropicales, las pérdidas en diferentes cultivos han superado el 45\%, ascendiendo hasta 77\% (APS 1975, Pineda \& Avila 1993).

Tomando en cuenta que ambos patógenos provienen del suelo, puede que algunas de las estrategias que se han aplicado (Ramírez et al 1998, Ortiz 2007), no sean totalmente efectivas, sobre todo si se aplican de forma individual, por lo que se hace necesario evaluarlas de manera combinada o experimentar nuevas alternativas. Por lo anterior, el objetivo de la presente investigación fue evaluar estrategias de tipo químico, físico, biológico y cultural solas o combinadas en la incidencia de la pudrición basal, la pudrición carbonosa y el rendimiento de Phaseolus vulgaris cv Tacarigua en una parcela en Sabaneta estado Barinas en Venezuela.

\section{Materiales y métodos}

Establecimiento del ensayo. El experimento se estableció entre Octubre 2007 y Febrero 2008, en un área de $360 \mathrm{~m}^{2}$, ubicada en el municipio Alberto Arvelo Torrealba, en Sabaneta estado Barinas a 165 msnm, en la antigua Estación del Instituto Nacional de Investigaciones Agrícolas (INIA) con una temperatura media anual para el año 2007 de $26.8{ }^{\circ} \mathrm{C}$ y una humedad relativa promedio de $77 \%$ (de acuerdo a datos suministrados por la estación climatológica del INIA), con un alto grado de infestación natural con Sclerotium rolfsii y Macrophomina phaseolina, de acuerdo a lo observado por los técnicos de la institución, en ciclos anteriores. La parcela, la cual fue sembrada de caraota en el ciclo 
anterior, se dividió en 36 sub-parcelas de $10 \mathrm{~m} \mathrm{x}$ $1 \mathrm{~m}$ y en cada una se conformaron dos hileras con semillas de caraota $P$. vulgaris cv Tacarigua en sistema de camellones del mismo suelo, levantados unos $25 \mathrm{~cm}$, con una distancia entre plantas promedio de $10 \mathrm{~cm}$., siendo la distancia tradicionalmente usada por los productores.

Tratamientos. Los tratamientos aplicados fueron: Solarización (Sol), Trichoderma harzianum (Tri), nitrato de calcio, producto importado con $11.5 \%$ de nitrógeno y 23\% de $\mathrm{CaO}$ ) [Ca], Tebuconazole (Teb) y las combinaciones SolTri, SolCa, SolTeb, TriCa y TebCa, así como un Testigo, sin la aplicación de los tratamientos. El 12 de octubre de 2007, se aplicó la solarización durante 15 días; para ello se cubrió el suelo con plástico transparente antes de la siembra, con un grosor aproximado de $3 \mathrm{~mm}$. Tres días después de culminada la solarización en los tres tratamientos bajo esta condición, se sembraron las semillas de caraota en toda la parcela experimental.

En aquellos tratamientos con Tri (Producto comercial Subiol $^{\circledR}: 1 \times 10^{12}$ esporas $/ \mathrm{mL}$ ), el mismo se mezcló con las semillas antes de la siembra, aplicando $15 \mathrm{~g}+3 \mathrm{~mL}$ de surfactante (Surfactron $^{\circledR}$ ) por cada $1.5 \mathrm{k}$ de semilla. La semilla fue proporcionada por un productor cooperante. Posteriormente, el antagonista se aplicó al suelo a los 15, 30, 45 y 60 días después de la siembra (dds), utilizando en cada oportunidad $30 \mathrm{~g}$ por cada $10 \mathrm{~L}$ de agua apta para consumo humano, cubriendo los tres tratamientos respectivos donde se aplicó el hongo benéfico. El nitrato de calcio (con un grado de pureza de 99\%) se aplicó al suelo a razón de 60 g por cada $10 \mathrm{~L}$ de agua a los cuatro tratamientos con el fertilizante.
Durante el desarrollo del cultivo, se realizaron 4 aplicaciones del insecticida Thionil $^{\circledR}, 2$ con el herbicida Gramoxone ${ }^{\circledR}+3$ limpiezas manuales, realizadas entre camellones, así como 2 aplicaciones de urea (46\% de nitrógeno, de la empresa estatal Pequiven) (el equivalente a 100 $\mathrm{k} / \mathrm{ha}$, en cada caso, dosis utilizada normalmente por los productores de la zona). Así mismo, se realizó análisis de suelo tomando muestras antes de la aplicación de alguna de las prácticas bajo estudio, para conocer la textura, cantidad de macroelementos (nitrógeno, fósforo y potasio) y el pH. La cosecha se realizó a los 93 días después de la siembra.

Desde de la siembra hasta la cosecha (30 de Octubre 2007 a 1 de Febrero de 2008) se registró la precipitación (PP: mm), la temperatura (TS: ${ }^{\circ} \mathrm{C}$ ) y la humedad absoluta (HA: $\mathrm{g} / \mathrm{m}^{3}$ ) del suelo. Los datos de PP de la zona fueron obtenidos de la estación climatológica ubicada en el campo experimental, mientras que la TS y la HA se obtuvieron a través de dispositivos electrónicos tipo datalogger marca $\mathrm{HOBO}^{\circledR}$ colocados a una profundidad en el suelo entre 0 y $15 \mathrm{~cm}$.

Variables evaluadas. Cuantitativamente se determinó el porcentaje de plantas enfermas (PPE), el área bajo la curva del progreso tanto de la pudrición basal como de la pudrición carbonosa $\left(\mathrm{ABCPE}_{\mathrm{T}}\right)$, así como el tiempo de inicio de la enfermedad $\left(\mathrm{T}_{0}\right)$. De manera cualitativa se evaluó la severidad de la pudrición carbonosa, tomando en cuenta el avance o no de las manchas hasta las vainas y las semillas. Así mismo, se registró el número de vainas vacías/planta (VVP), número de vainas con semillas/planta (VCS) y el rendimiento del cultivo (REN: k/ha). 
Diseño y análisis estadístico de los datos. Las unidades experimentales se dispusieron en un diseño en bloques al azar. Cada tratamiento constó de 3 repeticiones. Se realizó análisis de varianza y cuando fue necesario prueba de comparación de medias de acuerdo a Tukey. El programa utilizado fue el Statistical Analysis System 1995.

\section{Resultados}

Dinámica temporal e incidencia de la pudrición basal causada por Sclerotium rolfsii. El micelio de $S$. rolfsii se observó en la superficie del suelo, a partir de los 16 días después de la siembra (dds), siendo confirmado con la posterior producción de esclerocios de color marrón claro, los cuales son característicos de este patógeno (APS 1975). En cuanto a los síntomas, las plántulas mostraron una lesión marrón alrededor de la base del tallo, produciendo marchitez y posterior muerte, indicando la presencia de la pudrición basal (PB) inducida por este patógeno (APS 1975).

Los primeros tratamientos donde se detectaron los signos y síntomas de la PB fueron Ca y CaTri (16 dds); siendo en SolTeb, donde se observó el mayor retraso (42 dds). En Teb, TebCa y SolCa, se evidenció a partir de los 35 dds; en Tri a los 27 dds y en Test, Sol y SolTri a los 22 dds (Tabla 1). De esta forma, el fungicida solo o combinado con el nitrato de calcio o la solarización, evidenció un efecto importante al retrasar la acción del inóculo primario de $S$. rolfsii. Sin embargo, la curva del progreso de la pudrición basal observada en el testigo y el resto de los tratamientos hasta los 50 dds, mostró un comportamiento exponencial (Figura 1A y 1B) indicando la actuación tanto del inóculo primario como del secundario, aún en SolTeb; lo que puso de manifiesto la alta capacidad de sobrevivencia de $S$. rolfsii.

Tabla 1 Efecto de los tratamientos en el tiempo de inicio $\left(\mathrm{T}_{\mathrm{o}}\right.$ ) de la pudrición basal (PB), el porcentaje de plantas enfermas (PPE) por PB y el área bajo la curva del progreso de las enfermedades en forma total ( ABCPE $_{\mathrm{T}}$ : pudrición basal+pudrición carbonosa)

\begin{tabular}{lcll}
\hline TRATAMIENTOS & T $_{\mathbf{0}}$ (dds) & \multicolumn{1}{c}{ PPE/PB } & ABCPE $_{\mathbf{T}}$ \\
\hline Testigo (Test) & 22 & $14.5 \mathrm{ab}$ & $28.039 \mathrm{ab}$ \\
Trichoderma (Tri) & 27 & $28.5 \mathrm{a}$ & $34.465 \mathrm{a}$ \\
Solarización (Sol) & 22 & $21.6 \mathrm{ab}$ & $29.005 \mathrm{ab}$ \\
Calcio (Ca) & 16 & $15.4 \mathrm{ab}$ & $28.702 \mathrm{ab}$ \\
Tebuconazole (Teb) & 35 & $5.3 \mathrm{~b}$ & $22.859 \mathrm{~b}$ \\
SolTri & 22 & $10.1 \mathrm{ab}$ & $27.365 \mathrm{ab}$ \\
SolCa & 35 & $7.2 \mathrm{~b}$ & $23.433 \mathrm{ab}$ \\
SolTeb & 42 & $3.8 \mathrm{~b}$ & $21.461 \mathrm{~b}$ \\
TriCa & 16 & $22 \mathrm{ab}$ & $31.076 \mathrm{ab}$ \\
TebCa & 35 & $11.8 \mathrm{ab}$ & $26.284 \mathrm{ab}$ \\
\hline $\mathrm{r}^{2}$ & & 0.65 & 0.67 \\
CV & & 48.2 & 14.06 \\
\hline
\end{tabular}

dds: días después de la siembra de la caraota. Valores con letras iguales, tienen comportamientos similares de acuerdo a Tukey $(P \leq 0.01)$

El tratamiento Tri fue el que mostró el mayor porcentaje de plantas enfermas por PB (28.5\%), superando al testigo (Test) que alcanzó 14.5\% (Tabla 1, Figura 1A). El menor porcentaje correspondió a SolTeb (3.8\%) (Tabla 1, Figura 1B). Con el inicio de la formación de vainas y semillas, alrededor de los 50 días después de la siembra (dds), predominaron lesiones de color negro en la base del tallo de las plantas, las cuales fueron ascendiendo de forma discontinua, hacia las ramas, siendo característico de la pudrición carbonosa (APS 1975), daño inducido por Macrophomina phaseolina. 
Fig. 1A Curvas del progreso de las enfermedades pudrición basal (alrededor de los 50 días) y la pudrición carbonosa (posterior a los 50 días) en diferentes tratamientos aplicados en una parcela de caraota en Sabaneta estado Barinas. Período: Octubre 2007Febrero 2008.

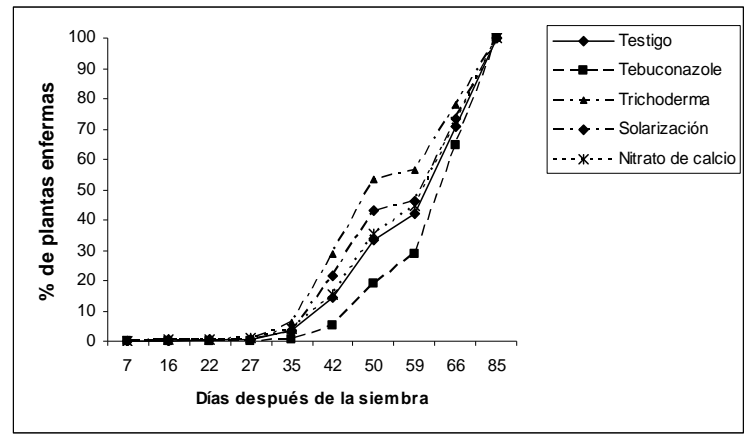

Fig. 1B

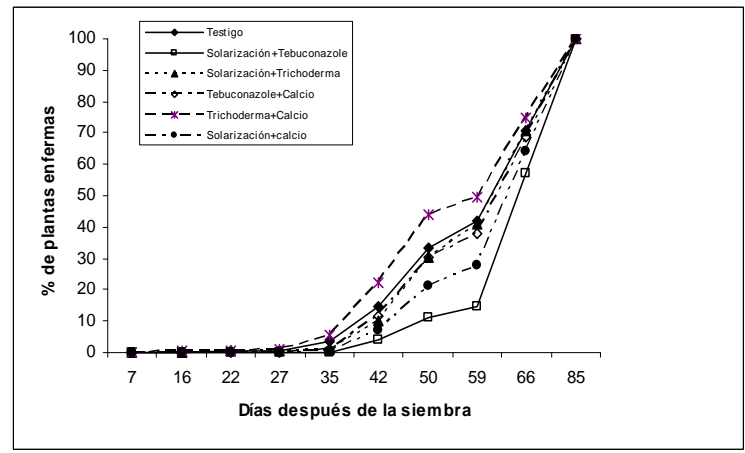

Dinámica temporal, incidencia y severidad de la pudrición carbonosa causada por Macrophomina phaseolina. A partir de los 50 dds, se inició el registro de plantas enfermas por la pudrición carbonosa (PC) inducida por el hongo M. phaseolina. A los 59 dds se evidenció un punto de inflexión en cada una de las curvas del progreso de la enfermedad, en todos los tratamientos, así como un ascenso completamente lineal en la incidencia de la pudrición carbonosa, completando el $100 \%$ de plantas enfermas en todo el ensayo causado por la co-ocurrencia de $S$. rolfsii y M. phaseolina.
En cuanto al área bajo la curva del progreso total de ambas enfermedades $\left(\mathrm{ABCPE}_{\mathrm{T}}\right)$ las menores correspondieron a los tratamientos SolTeb (21.461) y Tebuconazole (Teb: 22.859) siendo significativamente menores a Tri (34.465) que resultó ser la mayor, no obstante, todos estos tratamientos resultaron similares al Test (27.365).

Cualitativamente, hubo diferencias en la severidad de la pudrición carbonosa, al observar tanto la intensidad de la coloración negruzca, como el avance de la enfermedad, desde la base del tallo hasta las vainas, e inclusive en el manchado de las semillas. Aunque todas las plantas en los tratamientos mostraron síntomas de la pudrición carbonosa, a diferencia de lo detectado con la pudrición basal, en Solarización (Sol), Tebuconazole (Teb), SolTeb y SolTri, se evidenció menor cantidad de manchas tanto en la superficie del tallo y las ramas, como en las vainas al momento de la cosecha, en comparación con el resto de los tratamientos. En este sentido, la mayor severidad de la PC se observó en Ca, Tri, CaTri y el Test, en los cuales el manchado inducido por $M$. phaseolina cubrió toda la planta y la superficie de las vainas, afectando gran parte de las semillas. Estos resultados se reflejaron en el peso de estas últimas y por ende, en el rendimiento del cultivo.

Es importante resaltar, la presencia de abundantes nódulos característicos de la simbiosis entre rizobacterias y las plantas de caraota, de forma natural en el Test, los tratamientos solarizados y/o los de Trichoderma; sin embargo, no hubo formación de éstos, en aquellos tratamientos donde se aplicó Tebuconazole (Teb, TebCa y SolTeb) y nitrato de calcio en forma individual (Ca); aunque en la combinación de este fertilizante 
con la solarizaciòn y/o el antagonista, sí se evidenció la presencia de algunas de estas estructuras fijadoras de nitrógeno (datos no cuantificados).

Fig. 2 Comportamiento de la precipitación, la temperatura y la humedad absoluta del suelo en el ciclo de desarrollo del cultivo de caraota en una parcela de la antigua estación experimental del INIA en Sabaneta estado Barinas. Período: Octubre 2007-Febrero 2008.

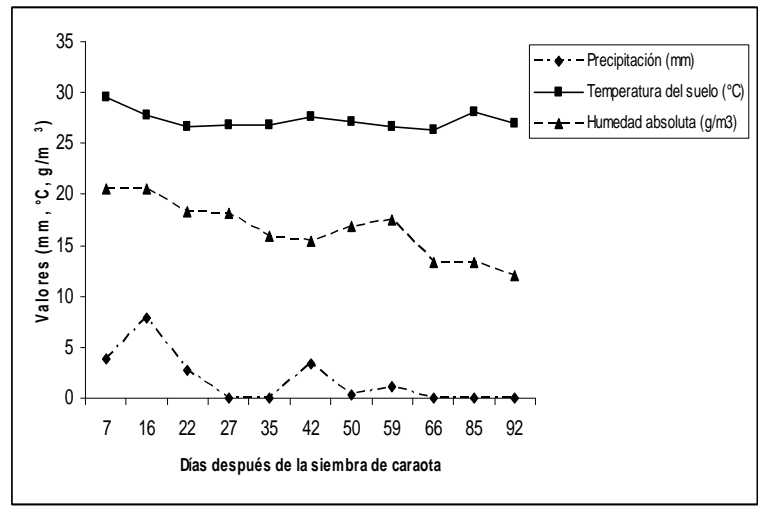

Relación de la condiciones de precipitación, temperatura y humedad absoluta del suelo con la aparición de la pudrición basal y la pudrición carbonosa. En la Figura 2 se observa el comportamiento de la precipitación (PP), la temperatura y la humedad absoluta del suelo (TS y HA, respectivamente), durante el ciclo de desarrollo de la caraota desde la primera semana de siembra hasta la cosecha. La PP osciló en promedio entre 0 y $7.84 \mathrm{~mm}$, entre intervalos de evaluación de las enfermedades bajo estudio, detectándose el mayor pico en la etapa de plántula, justo cuando se evidenció la presencia de $S$. rolfsii (día 16). Posterior a esta fecha inició el descenso y aunque mostró dos picos más de lluvia (días 42 y 59), éstos fueron menores (3.44 mm y $1.16 \mathrm{~mm}$ ). En general, la PP acumulada de los meses
Octubre, Noviembre y Diciembre (período que abarcó el ciclo del cultivo) fueron $76 \mathrm{~mm}, 123 \mathrm{~mm}$ y $42 \mathrm{~mm}$, respectivamente, para un total de 241 mm de lluvia.

La HA entre intervalos de evaluación en el ensayo, osciló en el suelo entre $12.1 \mathrm{~g} / \mathrm{m}^{3}$ y $20.5 \mathrm{~g} / \mathrm{m}^{3}$, siendo directamente proporcional a la PP, tal como se evidencia en la Tabla 3. En el caso de la TS, la misma disminuyó de $29.6{ }^{\circ} \mathrm{C}$ en la primera semana de cultivo, a $27.7{ }^{\circ} \mathrm{C}$ hasta el día 16 , tiempo en el cual se realizó la primera evaluación de la pudrición basal, observándose abundante micelio y la formación de esclerocios de $S$. rolfsii. A partir de ese momento y hasta los 66 dds, se detectó poca variación de la temperatura, manteniéndose entre 26.4 y $27.7^{\circ} \mathrm{C}$. Después de este período, se observó un incremento de la temperatura, alcanzando un promedio de $28.05^{\circ} \mathrm{C}$ entre los 66 y los 85 dds, coincidiendo con la sequía de las últimas semanas del cultivo, marcando el descenso de la HA (Figura 2) y la mayor virulencia de $M$. phaseolina.

Rendimiento de la caraota. En la Figura 3 se observa el rendimiento de la caraota ( $\mathrm{k} / \mathrm{ha})$, donde se pueden evidenciar 3 grupos entre los tratamientos. El primer grupo conformado por Teb y SolTeb; el segundo integrado por Sol, SolTri, SolCa, TebCa, Ca, Tri y el Test y el tercero por TriCa. Los dos primeros tratamientos fueron estadísticamente diferentes al último. El mayor rendimiento correspondió a Teb con 555 k/ha, mientras que el menor se registró en TriCa con 35 $\mathrm{k} / \mathrm{ha}$.

El mejor desarrollo en las plantas se observó en aquellas establecidas en tratamientos solarizados 
(TS), tales como Sol, SolTri y SolTeb, pero éstas también fueron las que presentaron la mayor cantidad de vainas vacías/planta (en promedio entre 11 a 18) (Tabla 2) aunque, de acuerdo a la prueba de Tukey no hubo diferencias significativas $(P>0.05)$ para esta variable.

Fig. 3 Rendimiento de la caraota (k/ha) en los diferentes tratamientos en una parcela experimental en Sabaneta estado Barinas (Ciclo Octubre 2007- Enero 2008). Valores con letras iguales, tienen comportamientos similares de acuerdo a Tukey $(P \leq 0.01) . r^{2}: 0,66$ CV: 50,8.

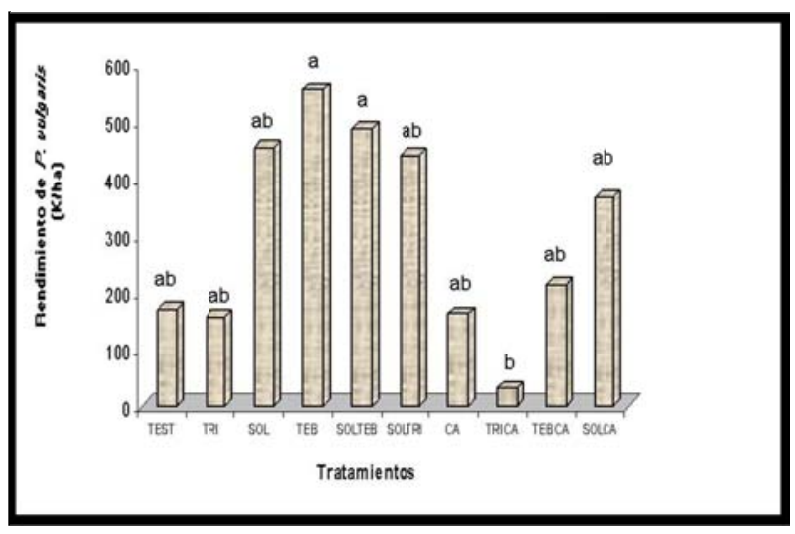

Hubo un hecho adicional al efecto negativo que causó la presencia de ambas enfermedades, referido a un retraso en el ciclo del cultivo. Cercano a los 75 días, mientras las plantas del testigo y otros tratamientos sin solarización, habían culminado su madurez fisiológica, alcanzando el secado natural de las hojas, aquellos tratamientos solarizados, mantuvieron su cubierta foliar completamente verde pasados los 85 dds. Esta situación repercutió en el rendimiento del producto final, bien por una mayor producción de vainas vacías (en Sol, SolTeb y SolTri) o por la poca producción de semillas/vaina (SolCa).
Tabla 2 Efecto de los tratamientos en la cantidad de vainas con semillas (VCS) y la formación de vainas vacías por planta (VVP).

\begin{tabular}{ccc}
\hline TRATAMIENTOS & VCS & VVP \\
\hline Testigo (Test) & $5.3 \mathrm{~b}$ & $8.0 \mathrm{a}$ \\
Trichoderma (Tri) & $9.5 \mathrm{ab}$ & $11.0 \mathrm{a}$ \\
Solarización (Sol) & $14.3 \mathrm{a}$ & $11.0 \mathrm{a}$ \\
Calcio (Ca) & $8.8 \mathrm{ab}$ & $5.3 \mathrm{a}$ \\
Tebuconazole (Teb) & $11.8 \mathrm{ab}$ & $8.7 \mathrm{a}$ \\
SolTri & $12.8 \mathrm{ab}$ & $13.3 \mathrm{a}$ \\
SolCa & $7.5 \mathrm{ab}$ & $3.7 \mathrm{a}$ \\
SolTeb & $6.9 \mathrm{ab}$ & $18.0 \mathrm{a}$ \\
TriCa & $5.5 \mathrm{~b}$ & $3.5 \mathrm{a}$ \\
TebCa & $7.5 \mathrm{ab}$ & $5.7 \mathrm{a}$ \\
\hline $\mathrm{r}^{2}$ & 0.64 & 0.59 \\
CV & 33.3 & 45.8 \\
\hline Valores con letras iguales, & tienen comportamientos & similares de \\
acuerdo a Tukey. & &
\end{tabular}

En el caso de las vainas con semillas (VCS) [no necesariamente vainas llenas], las mismas oscilaron en promedio entre 5.3 a 14.3/tratamiento. Sol resultó con el mayor número de VCS, siendo estadísticamente diferente a TriCa y al Test (Tukey; $P<0.05$ ) (Tabla 2). Sin embargo, las semillas más grandes y uniformes, así como el mayor número de semillas/vaina (NSPV), se observaron en Teb y SolTeb; en el resto de los tratamientos el tamaño de la semilla y el NSPV fue variable (datos no mostrados), reflejándose en el rendimiento.

\section{Discusión}

Tanto en el testigo como en el resto de los tratamientos, la forma de la curva de las enfermedades evaluadas los primeros 50 días (período de registro de la pudrición basal) y posteriormente hasta los 85 días (registro de la pudrición carbonosa) evidenciaron 
comportamientos propios de enfermedades policíclicas. Esto implicó la actuación, tanto de los esclerocios de Sclerotium rolfsii y Macrophomina phaseolina establecidos antes de la siembra de la caraota, como de micelio y nuevos esclerocios producidos por estos hongos, diseminados planta a planta a través de las raíces.

En este sentido se puede afirmar, que ninguno de los tratamientos debió disminuir de manera significativa las poblaciones iniciales de esclerocios de los patógenos involucrados, tomando en cuenta que se produjo más de un ciclo de infección, característico de enfermedades policíclicas. No obstante, alternativas como el fungicida Tebuconazole aplicado solo o combinado con la solarización, sí lograron la disminución de parámetros epidemiológicos, tales como el área bajo la curva del progreso de las enfermedades evaluadas, la incidencia final de la pudrición basal causada por Sclerotium rolfsii y el nivel de virulencia de $M$. phaseolina (evidenciado a través del avance de las lesiones desde la base del tallo hasta las vainas) al compararlo con el testigo y tratamientos con Trichoderma harzianum y/o nitrato de calcio. Resultados similares en tratamientos con Tebuconazole o solarización, han sido señalados por Delgadillo et al 2002, Ulacio et al 2006, respectivamente, en la pudrición blanca del ajo inducida por Sclerotium cepivorum Berk.

El fungicida, a pesar de que impidió la formación de nódulos propios de bacterias fijadoras de nitrógeno, no pareció influir en el crecimiento de las plantas (al compararlo con el resto de los tratamientos), siendo la alternativa que produjo 3.36 y 15.85 veces más kilogramos de semillas, en comparación con el testigo y el tratamiento TriCa, respectivamente. De esta forma, la planta fue capaz de tolerar el ataque de los patógenos objetos de estudio. No obstante, la ausencia de la fijación simbiótica rizobacteria-raíces, producto de la aplicación del agroquímico tóxico Tebuconazole, debió interferir en la obtención de una mayor cantidad de semillas de caraota, por la función que cumplen los nódulos formados por la bacteria en la obtención de nitrógeno atmosférico, importante para la producción de este cultivo (Morros 2001).

Resultados de la inhibición de la nodulación por rizobacterias en leguminosas, como consecuencia del uso de productos del grupo de los Triazoles, ha sido señalado en soya por Faessel et al 2010. Estos investigadores comprobaron que el producto Acibenzolar-S-metil (ASM) inhibió la formación de nódulos por parte de Bradyrhizobium japonicum en raíces, coincidiendo con los resultados obtenidos con Tebuconazole. No obstante, Faessel et al 2010 señalaron que la falta de simbiosis bacteria-soya, no puede ser explicada por efectos directos antimicrobiales del ASM, debido a que en su investigación, la tasa de crecimiento de $B$. japonicum en un medio que contenía este producto, fue similar al del control.

Sin embargo, es posible que el retraso en la virulencia de $M$. phaseolina evidenciado en la menor severidad en las vainas y semillas de caraota, en tratamientos con Tebuconazole utilizado en Barinas Venezuela, haya sido producto de una respuesta de resistencia sistémica adquirida (RSA) como consecuencia del uso del fungicida. En este sentido, Faessel et al 2010 comprobaron que el uso de ASM redujo la 
severidad de los síntomas de la pudrición causada por Rhizoctonia solani en plantas de soya, producto de la inducción de RSA (Faessel et al 2008), lo que explicaría los resultados del ensayo en caraota.

La Solarización (Sol) no alcanzó temperaturas letales continuas (mayores a $40{ }^{\circ} \mathrm{C}$ por varias horas en el día) como para afectar la viabilidad de los esclerocios de $S$. rolfsii y $M$. phaseolina, tal como ha ocurrido con $S$. cepivorum en ajo (Ulacio et al 2006); por el contrario, en el experimento con caraota, los patógenos fueron favorecidos en su efecto fitopatológico con temperaturas que oscilaron entre 28 a $33^{\circ} \mathrm{C}$ en el suelo solarizado (SS) cuando esta alternativa se implementó de manera individual, tal como se evidenció con la incidencia de plantas enfermas. Las temperaturas alcanzadas en el SS fueron similares a las registradas en el suelo no solarizado (SNS). Este fracaso en la solarización al no alcanzar temperaturas al menos sub-letales de manera continua (entre $30{ }^{\circ} \mathrm{C}$ y $40{ }^{\circ} \mathrm{C}$, según Tjamos \& Fravel 1995) fue consecuencia del período en el cual se implementó, dado que hubo pocos días soleados.

La temperatura que se mantuvo en el SS logró eliminar las malezas de hoja ancha, pero no la de hoja angosta, por lo que el suelo mantuvo una cubierta vegetal, que impidió el aumento de la temperatura, a aquellas consideradas sub-letales (entre 30 y $40{ }^{\circ} \mathrm{C}$ ) mantenidas por unas $10 \mathrm{~h}$. continuas en un día que pudieron retrasar la germinación de los esclerocios, tal como ha ocurrido con microesclerocios de Verticillium dahliae expuestos a temperaturas de 31 a $38{ }^{\circ} \mathrm{C}$ por 10 a 14 h días continuas (Tjamos \& Fravel
1995) o que pudieron haber debilitado a los esclerocios para ser presa fácil de los saprófitos presentes en el suelo (entre ellos, Aspergillus spp, Penicillium spp o Rhizopus spp), tal como lo ha señalado Katan 1981, Ulacio et al 2006.

A pesar de que al final del ciclo del cultivo, el $100 \%$ de las plantas que quedaron resultaron enfermas por $M$. phaseolina, desde el punto de vista de manejo de las enfermedades, SolTeb se constituyó en el tratamiento que logró retrasar tanto la incidencia de la pudrición basal, como la severidad de la pudrición carbonosa.

En el caso de Trichoderma harzianum, son conocidos no solo los beneficios de su aplicación, como antagonista en sus funciones de hiperparasitismo, competencia o antibiosis (Ezziyyani et al 2004, Ulacio et al 2002) o como inductor de crecimiento y de resistencia en las plantas (Yedidia et al 1999), sino también que se ha comprobado su compatibilidad con rizobacterias, cuando se programa su aplicación (Bécquer 2004). Sin embargo, fue indudable que las condiciones dadas en el patosistema evaluado, no favorecieron la acción benéfica que se le ha comprobado a este hongo. Estos resultados coinciden con Cardona \& Rodríguez 2002, Pineda \& Gonnella 1988, quienes comprobaron que la habilidad saprofítica competitiva de $T$. harzianum que fue igualada por M. phaseolina en ajonjolí con respecto a un testigo (16\%) al observarse mayor cantidad de plantas muertas (Vivas 2006) quien evidenció mayor número de semillas de 
caraota dañadas por $S$. rolfsii (12\%) donde se aplicó el antagonista, en comparación con un control que alcanzó 8.6\%.

La aplicación de nitrato de calcio, a pesar de que fueron los tratamientos con menor número de vainas vacías, también fueron los que produjeron la menor cantidad con semillas y aunque aplicado solo inhibió la formación de nódulos por rizobacterias, combinado con Trichoderma o la solarización, sí pudo producirlos. Una explicación en la falta de nodulación, pudiera coincidir con lo señalado por Mendez 2010. Según este autor, la simbiosis de bacterias con las raíces de las plantas, se inhibe en presencia de un alto contenido de nitrógeno en forma de nitrato o amonio, a pesar que el calcio juega un papel preponderante en el inicio de los nódulos. Esto indica la necesidad de seleccionar para el cultivo de caraota, una fuente de calcio sin nitrógeno para que la fijación simbiótica de las rizobacterias no sea neutralizada.

Sin embargo, la presencia de Trichoderma y/o la solarización combinado con el fertilizante, debió estimular la actividad entre los microorganismos presentes en el suelo, metabolizando gran parte del nitrato de calcio aplicado, lo que permitió la interacción de la bacteria con las raíces de las plantas de caraota, donde se aplicaron estas estrategias de manejo, permitiendo la formación de algunos nódulos. En contraste, el referido nitrato de calcio, no presentó algún efecto positivo adicional en la leguminosa, tal como sí se ha comprobado en ajo donde ha contribuido en el engrosamiento de la pared y el aumento en el área celular de la raíz, limitando a la pudrición blanca, enfermedad causada por $S$. cepivorum (Rojas et al 2009).

Los resultados anteriores en cuanto a la incidencia de las enfermedades confirman que efectivamente la aplicación de Trichoderma solo o combinado con nitrato de calcio (TriCa) es contraproducente en las plantas de caraota. Al parecer, el antagonista, no solo potenció la habilidad parasítica competitiva de $S$. rolfsii y $M$. phaseolina, sino que debió competir con la rizobacteria fijadora de nitrógeno al momento de la formación de nódulos en las raíces, neutralizándose la acción del biocontrolador (Bécquer 2004).

Evidentemente, el inicio del ciclo patogénico tanto de $S$. rolfsii como de M. phaseolina en las plantas de caraota, fue determinado tanto por la temperatura como la humedad del suelo, tal como lo han señalado Ferreira \& Boyle 2005 para $S$. rolfsii, Cardona et al 1997, Cardona 2006, Cardona \& Rodríguez 2002, para M. phaseolina. La disminución paulatina de la precipitación (PP) después de los 42 días de desarrollo del cultivo (12 de Diciembre de 2007) coincidió con la aparición de los síntomas de la pudrición carbonosa (PC) inducida por M. phaseolina, desplazando casi por completo la presencia de los propágulos de $S$. rolfsii.

Dada las variaciones mostradas en la PP, en comparación con lo detectado con la temperatura del suelo (TS) en este ensayo (Fig. 2), fue indudable que la cantidad de lluvia tuvo mayor influencia que la temperatura del suelo, en la patogénesis de ambos hongos. En este sentido, la mayor sequía determinó la aparición de $M$. 
phaseolina, coincidiendo con lo señalado por Cardona 2006, Cardona et al 1997. Así mismo, la cantidad de agua acumulada en el suelo determinó también, el éxito o fracaso de algunos tratamientos en el avance o no de la severidad y agresividad de la PC, siendo más intenso en plantas del Test y los tratamientos Ca, Tri y TriCa después de los 59 días de desarrollo del cultivo, tiempo en el cual la humedad absoluta (HA) descendió por debajo de $15 \mathrm{~g} / \mathrm{m}^{3}$ de suelo (Figura 2).

\section{Conflictos de interés}

Esta investigación recibió financiamiento total CDCHT /UCLA Lara, Venezuela, y no presenta conflictos de interés.

\section{Agradecimientos}

Los autores agradecen al Consejo De Desarrollo Científico Humanístico y Tecnológico (CDCHT) de la Universidad Centroccidental "Lisandro Alvarado" por el financiamiento de la presente investigación bajo el código 009 AG-2005.

\section{Literatura citada}

Acevedo F. El cultivo de la caraota. Fondo editorial UNELLEZ. Ediciones de la Universidad Ezequiel Zamora. 2003;208 pp.

American Phytopathological Society (APS). Compendium of Soybean diseases. J. Sinclair and M. Shurtleff (co-editors). St Paul, Minnesota. USA. 1975;69 pp.

Bécquer C, Ramos Y, Nápoles J, Arioza M. Efecto de la interacción Trichoderma24 rizobio en Vigna luteola SC-123. Pastos y Forrajes. 2004;27:139-145.

Cardona R. Distribución vertical de esclerocios de Macrophomina phaseolina en un suelo infestado naturalmente en el estado Portuguesa. Revista de la Facultad de Agronomía (LUZ). 2006;23:284-291.

Cardona R, Rodríguez H. Evaluación de Trichoderma harzianum en el control de Macrophomina phaseolina en ajonjolí. Fitopatología Venezolana. 2002;15:21-23.

Cardona R, Rodríguez H. Efecto del hongo Trichoderma harzianum sobre la enfermedad pudrición carbonosa en ajonjolí. Revista de la Facultad de Agronomía (LUZ). 2006;23:42-48.

Cardona R, Rodríguez H, Nass H. Dinámica poblacional de microesclerocios de Macrophomina phaseolina en un suelo naturalmente infestado bajo rotación de cultivo. Fitopatología Venezolana. 1997;11:23-26.

Delgadillo F, Zavaleta E., Osada S, Arévalo A, González V, Nieto D, Torres I. Densidad de inóculo de Sclerotium cepivorum y su control mediante Tebuconazole en ajo (Allium sativum L.). Revista Fitotecnia Mexicana. 2002;25:349354.

Ezziyyani M, Pérez C, Sid A, Requena M, Candela M. Trichoderma harzianum como biofungicida para el control de Phytophthora capsici en plantas de pimentón (Capsicum annuum L.). Annales de Biología. 2004;26:35-45. 
Faessel L, Nassr T-Lebeau N, Walter B. Effects of the plant defense inducer acibenzolar-S-methyl on hypocotyls rot of soybean caused by Rhizoctonia solani AG4. Journal Phytopathology. 2008;156:236242.

Faessel L, Nassr, T-Lebeau N, Walter B. Chemically-induced resistance on soybean inhibits nodulation and mycorrhization. Plant soil. 2010;329:259-268.

Ferreira S, Boyle R. Patología de plantas. Universidad de Hawai 2005. Disponible: http://www.extenso.Hawaii./KBase//crop/t ype(srofs.htlm.24k)

Katan J. Solar heating (solarization) of soil for control of soilborne pest. Annual Review of Phytopathology. 1981;19:211-236.

Mendez F. Efecto de una bacteria tipo Rhizobium en jitomate saladette. 2010. Disponible: http://www.scribd.com.

Mora O. Origen e importancia del cultivo de la caraota (Phaseolus vulgaris L.). Revista de la Facultad de Agronomía (Maracay). 1997;23:225-234.

Morros M. Cultivo de la caraota con énfasis en el estado Lara. Instituto Nacional de Investigaciones Agrícolas. Centro de Investigaciones Agropecuarias del Estado Lara. Serie D. $N^{\circ}$ 43. Barquisimeto estado Lara. 2001;74 pp.

Ortíz D. Enmiendas orgánicas y Trichoderma harzianum Rifai como estrategias para el control de la pudrición basal causada por Macrophomina phaseolina (Tassi) Goid. en Caraota (Phaseolus vulgaris L.). Trabajo
Especial de Grado. Universidad Centroccidental Lisandro Alvarado. Barquisimeto, Venezuela. 2007;38 pp.

Pineda J, Avila J. Alternativas para el control de Macrophomina phaseolina y Fusarium oxysporum, patógenos de ajonjolí (Sesamum indicum L.). Agronomía Tropical. 1993;38:79-84.

Pineda J, Gonnella E. Evaluación del control biológico de Macrophomina phaseolina en ajonjolí (Sesamum indicum L.). Agronomía Tropical. 1988;38:43-48.

Punja K. The biology, ecology and control of Sclerotium rolfsii. Annual Review of Phytopathology. 1985;3:97-127.

Ramírez R, Santos R, Bracho F, Sandoval L, Castro De Rincón C. Control de Sclerotium rolfsii Sacc. con fungicidas y humus. Revista de la Facultad de Agronomía (LUZ). 1998;15:534-544.

Rojas V, Ulacio D, Sanabria M, Jiménez M. Efecto del calcio, Trichoderma y brócoli en la pared y área celular del ajo para el control de la pudrición blanca. Boletín del Centro de Investigaciones Biológicas. 2009;43:183-196.

Statistical Analysis System Institute. SAS user's guide: Statistics version $6.4^{\text {th }}$ Edition. Cary. North Carolina. USA. 1995;956 pp.

Tjamos C, Fravel D. Detrimental effects of subletal heating and Talaromyces flavus on microsclerotia of Verticillium dahliae. Phytopathology. 1995;85:388-392. 
Ulacio D, Salas J, Querales P, Sanabria M. Micobiota del suelo de zonas productoras de papa del estado Mérida y su relación con Rhizoctonia solani. Bioagro. 2002;14:11-16.

Ulacio D, Zavaleta E, Martínez A, Pedroza A. Strategies for management of Sclerotium cepivorum Berk. in garlic. Journal of Plant Pathology. 2006;88:253-261.

Vivas D. Manejo de la pudrición basal por Sclerotium rolfsii Sacc. en caraota (Phaseolus vulgaris L.) incorporando al suelo gallinaza, calcio y Trichoderma harzianum. Trabajo Especial de Grado. Universidad Centroccidental Lisandro Alvarado. Barquisimeto, Venezuela. 2006;66 pp.

Yedidia B, Benhamou N, Chet I. Induction of defense responses in cucumber plants (Cucumis sativus L.) by the biocontrol agent Trichoderma harzianum. Applied and Environmental Microbiology. 1999;64:1061-1070. 